\title{
Differential Diagnoses of Inclusion Body Myositis (IBM)
}

Vinojini Vivekanandam, Enrico Bugiardini, Ashirwad Merve, Matthew Parton, Jasper M Morrow, Michael G Hanna, Pedro M Machado

\section{Authors' information}

1. Vinojini Vivekanandam

ORCID: 0000-0002-4944-0061

Address: Queen Square Centre for Neuromuscular Diseases, Department of Neuromuscular Diseases, University College London, $1^{\text {st }}$ Floor, Russell Square House, 10-12 Russell Square, WC1B 5EH London, UK Email: v.vivekanandam@ucl.ac.uk

Telephone number: +442031087182

2. Enrico Bugiardini

ORCID: 0000-0002-9709-6699

Address: Queen Square Centre for Neuromuscular Diseases, Department of Neuromuscular Diseases, University College London, 1st Floor, Russell Square House, 10-12 Russell Square, WC1B 5EH London, UK Email: e.bugiardini@ucl.ac.uk

Telephone number: +442031087521

\section{Ashirwad Merve}

Address: Department of Neuropathology, UCL Institute of Neurology, 1st Floor, Queen Square House, 22 Queen Square, WC1N 3BG London, UK

Email: amerve@nhs.net

Telephone number: +442034484239

4. Matthew Parton

Address: Queen Square Centre for Neuromuscular Diseases, Department of Neuromuscular Diseases, University College London, Ground Floor, 8-11 Queen Square, WC1N3BG London, UK 
Email: matt.parton@nhs.net

Telephone number: +442034488003

5. Jasper M Morrow

ORCID: 0000-0001-6879-8539

Address: Queen Square Centre for Neuromuscular Diseases, Department of Neuromuscular Diseases, University College London, 1st Floor, Russell Square House, 10-12 Russell Square, WC1B 5EH London, UK Email: j.morrow@ucl.ac.uk

Telephone number: +442034488259

6. Michael G Hanna

Address: Queen Square Centre for Neuromuscular Diseases, Department of Neuromuscular Diseases, University College London, Ground Floor, 8-11 Queen Square, WC1N3BG London, UK

Email:m.hanna@ucl.ac.uk

Telephone number: +442034488014

7. Pedro M Machado

ORCID: 0000-0002-8411-7972

Address: Queen Square Centre for Neuromuscular Diseases, Department of Neuromuscular Diseases; Centre for Rheumatology, Division of Medicine; University College London, $1^{\text {st }}$ Floor, Russell Square House, 10-12 Russell Square, WC1B 5EH London, UK

Email: p.machado@ucl.ac.uk

Telephone number: +442034488014 


\section{Corresponding author}

Pedro M Machado

Queen Square Centre for Neuromuscular Diseases

Department of Neuromuscular Diseases

University College London

1st Floor, Russell Square House

10-12 Russell Square

WC1B 5EH London, UK

Email: p.machado@ucl.ac.uk

Telephone number: +442031087515

\section{Disclosure statement}

PMM has received consulting/speaker's fees from Abbvie, BMS, Celgene, Eli Lilly, Janssen, MSD, Novartis, Pfizer, Roche and UCB.

\section{Acknowledgements}

Pedro M Machado is supported by the National Institute for Health Research (NIHR) University College London Hospitals (UCLH) Biomedical Research Centre (BRC). The views expressed are those of the authors and not necessarily those of the (UK) National Health Service (NHS), the NIHR, or the (UK) Department of Health.

\section{Keywords}

Inclusion Body Myositis; Muscular Dystrophy; Muscle Biopsy; Magnetic Resonance Imaging; Inflammatory Myopathy; Diagnosis. 


\section{Synopsis/Abstract}

Inclusion Body Myositis a slowly progressive myopathy, characteristically affecting quadriceps and long finger flexors. However atypical presentations do occur and there is overlap with other myopathies, including inflammatory and hereditary aetiologies. We discuss atypical cases, differential diagnoses and consider the role of imaging and histopathology in differentiating inclusion body myositis. 


\section{Key Points}

- Inclusion Body myositis (IBM) is a slowly progressive myopathy with characteristic early involvement of quadriceps and long finger flexors. However, less common presentations (e.g. dysphagia) are important to recognise.

- Clinical assessment complemented by neuromuscular Magnetic Resonance Imaging (MRI) is useful in choosing the optimal muscle to biopsy.

- Clinicopathological correlation is imperative in interpretation of the muscle biopsy and guiding further staining.

- MRI can also be useful in delineating patterns of muscle involvement to differentiate IBM from other primary muscle conditions, namely inherited myopathies.

- Inflammatory features on muscle biopsy can be seen in the idiopathic inflammatory myopathies (polymyositis, dermatomyositis, IBM and immune mediated necrotising myopathies) and in some inherited muscle conditions such as facioscapulohumeral muscular dystrophy (FSHD) and other genetic muscular dystrophies, particularly dysferlinopathies. 


\section{Introduction}

The term Inclusion Body Myositis (IBM) was first coined in 1971. A subsequent case series eight years later described the characteristic early pattern of weakness involving the quadriceps and long finger flexors ${ }^{1}$. Our understanding of the condition has since substantially improved.

IBM has an estimated prevalence of 139 per million above the age of 50 and an overall estimated prevalence of 46 per million ${ }^{2}$. While it is typically thought of as affecting those over 50 , approximately $20 \%$ develop symptoms in their forties ${ }^{3}$. There is a male predominance. Whites are more affected although the exact racial differences are not clear, as studies tend to cluster in the same geographic areas.

Although IBM is slowly progressive, there is currently no effective treatment and the resultant disability can be significant. Estimated health care costs are significant and several co-morbidities, including hypertension, hyperlipidaemia and ischaemic heart disease have been associated with IBM ${ }^{4}$. Using a Bayesian survival model for IBM, an increased risk of premature death compared with the general population of the same age and gender has recently been suggested for $\mathrm{IBM}^{5}$.

Together with the increased use of MRI, muscle biopsies and natural history studies, our understanding of pathogenesis has improved, leading to instigation of treatment trials. Treatment approaches that have been recently tested in IBM include the inhibition of the myostatin pathway with Follistatin gene therapy ${ }^{6}$ and Bimagrumab $^{7}$, and targeting protein dyshomeostasis with Arimoclomol ${ }^{8}$ and Rapamycin ${ }^{9}$. A large randomized, double-blinded, placebo-controlled phase 2 clinical trial of arimoclomol for the treatment of IBM is currently underway (NCT02753530).

Prevalence figures are probably underestimates because of the high rate of misdiagnosis. Moreover, there is a four to six year delay in diagnosis ${ }^{1}$. Accurate diagnosis is pivotal in providing prognostic information, support in the home as well as accessing clinical trials. We describe five cases that illustrate key diagnostic challenges and important differential diagnoses to consider. 


\section{CASE A: A lump in the throat.}

A 69 year old gentleman presented with an eight year history of swallowing difficulty. He had previously had a hemithyroidectomy for follicular adenoma and took amlodipine for hypertension. He lived with his wife on a farm. He was active and maintained his farm.

He first noticed difficulty with dry foods but went on to have difficulty with liquids, needing to 'gulp' them down and swallow several times. He was initially assessed by an Ear, Nose and Throat surgeon with no cause found and was referred to speech and language therapy. He was able to manage cut up solid foods but liquidised vegetables including beans and leeks.

Overtime he had increasing difficulty with solids, taking two hours to eat a meal. He unintentionally lost approximately eight kilograms over eight years. He coughed a lot when eating and had one chest infection over this period. He remained very active on his farm but had more recently noticed some difficulty climbing stairs.

He went on to have investigations including barium swallow, Magnetic Resonance Imaging (MRI) brain and cervical spine, electromyography, anti-cholinesterase and anti-MUSK antibodies, which were all nondiagnostic. An elevated Creatinine Kinase (CK) of $746 \mathrm{IU} / \mathrm{L}$ led to referral to a muscle specialist.

Examination at this stage demonstrated normal facial strength with normal eye movements. There was no fatigueable ptosis and no tongue wasting or fasciculations. In the upper limb, bulk was normal; however, there was subtle weakness of distal finger flexors. In the lower limbs, quadriceps muscle wasting was noted. Lower limb strength Medical Research Council (MRC) strength testing was normal (5) in all groups. However, he was unable to stand from a squat. Reflexes, sensory examination and co-ordination were all normal.

He went on to have lower limb MRI which demonstrated fatty infiltration of the anterior thigh compartment typical of IBM. Subsequent left vastus lateralis biopsy demonstrated features supportive of a diagnosis of IBM including prominent vacuoles, several Cytochrome C oxidase (COX) negative fibres and major histocompatibility complex class I (MHC Class I) upregulated p62 positive fibres.

He went on to have a percutaneous endogastric (PEG) tube inserted to supplement diet and boost nutrition. 


\section{Clinical Questions}

What percentage of patients with IBM present with dysphagia?

1. $25-50 \%$

2. $11-25 \%$

3. $4-10 \%$

4. $<4 \%$

What other neuromuscular conditions can present with dysphagia?

1. Myasthenia Gravis

2. Motor Neurone Disease

3. Chronic inflammatory demyelinating polyneuropathy

4. All of the above

\section{Discussion}

Dysphagia is an important feature to recognise as part of IBM. The prevalence of dysphagia varies between cohorts ranging from $46-86 \%$, however this tends to present later in the disease course ${ }^{10-13}$. A review of patients with IBM seen at the MRC Centre for Neuromuscular Diseases in 2013, found 4 of 51 patients (7.8\%) reported symptom onset was with dysphagia ${ }^{12}$. Similarly, $4.4 \%$ of patients first presented with swallowing difficulty in a cohort of 140 patients from two centres in Paris and Oxford ${ }^{14}$ and $9 \%$ of 64 patients in a Dutch cohort $^{13}$. Disease onset with dysphagia is rare but important to recognise in order to guide management, prevent aspiration and excessive weight loss.

Patients initially report dysphagia to solid foods followed by increasing difficulty with liquids and secretions ${ }^{15}$. The oropharyngeal weakness responsible for dysphagia may also cause obstructive sleep apnoea ${ }^{10}$.

Considering muscle diseases in the differential diagnosis for dysphagia is important. The diagnosis of IBM in particular is important to look for. Questions pertaining to characteristic muscle groups affected in IBM may be helpful: 
- Difficulty climbing stairs, rising from a low chair or the floor, which may suggest subtle proximal lower limb weakness.

- Difficulty with writing and fine motor tasks, and in particular, griping a pen.

These features may suggest early weakness in characteristic muscle groups affected in IBM. Clinical examination of these muscles in a patient presenting with dysphagia is also important:

- Long finger flexors (flexor digitorium profundus)

- Knee extension (quadriceps femoris)

- Hip flexion (iliopsoas, rectus femoris, proximal leg muscles)

- Knee extension is characteristically weaker than hip flexion.

As in our case, muscle MRI may additionally demonstrate characteristic patterns of involvement, which may be subtle and not clinically obvious.

Differential diagnoses for neuromuscular causes of dysphagia are important to appreciate and are illustrated in Table 1.

Management of dysphagia is important for patients with IBM. Assessment and recommendations from a Speech and Language Therapist include swallowing methods such as the Mendelsohn manoeuvre and dietary adjustment. Symptomatic improvement with cricopharyneal myotomy and pharyngoesophageal dilation is reported ${ }^{16}$. In more advanced disease, PEG insertion may be required. 


\section{CASE B: Third time lucky.}

A 64 year old gentleman presented with a three-year history of difficulty climbing stairs. His medical history is significant for spondylosis and varicose veins. He was retired but continued work as a Scouts Commissioner.

He first noticed difficulty after slipping while walking upstairs. Managing stairs became progressively more difficult, progressing to needing to use the handrail at all times. Getting out of low chairs also became difficult. He most recently noticed that he could no longer give the Scout salute at parades. He found that fingers on his right hands seemed to curl in. There were no other cranial nerve or sensory symptoms.

Electromyography was consistent with a myopathy and CK was 444 IU/L. However, investigations for inflammatory or immune causes of a myopathy were not diagnostic. He went on to have a muscle biopsy of the quadriceps, which demonstrated only fibrous tissue. A second biopsy of the left deltoid was performed but did not demonstrate any significant changes. Subsequent referral to a muscle specialist to investigate for other adult onset myopathies was made.

On examination, cranial nerves were normal. Upper limb examination demonstrated mild weakness of the distal finger flexors. In the legs, there was marked quadriceps wasting and severe knee extension weakness. Reflexes, sensation and co-ordination were normal.

Further review of the clinical history did not reveal any additional features, developmental delay or family history.

Given that proximal lower limb muscles were severely involved, while proximal upper limb muscles were unaffected, a distal forearm muscle, which was mild-moderately affected, was chosen to perform a repeat muscle biopsy.

Histopathology demonstrated features of IBM (Figure 1). The features include myopathic changes, rimmed vacuoles, upregulation of MHC Class I, p62+ inclusions, several COX deficient fibres, upregulation of MHC class I (HLA-ABC), chronic inflammatory cell infiltrate in the endomysium, p62+ inclusions and several fibres with COX deficiency. 


\section{Clinical Questions}

What is a differential diagnosis for an adult onset myopathy?

1. Duchene Muscular Dystrophy

2. Spinal Muscular Atrophy type 1

3. Pompe's Disease

4. Myotonia Congenita

Which factor is ideal for the choice of muscle to biopsy?

1. An atrophic muscle

2. A clinically weak muscle

3. A clinically strong muscle

4. A muscle normal on MRI

Do all IBM biopsies have rimmed vacuoles? Which statement is true?

1. $50 \%$ of biopsies have rimmed vacuoles

2. $15-20 \%$ of biopsies have rimmed vacuoles

3. $15-20 \%$ of biopsies do not have rimmed vacuoles

4. All muscle biopsies in IBM have rimmed vacuoles

\section{Discussion}

Other causes of myositis, such as dermatomyositis, polymyositis and immune-mediated necrotising myopathies, are often associated with a more acute onset and more rapid progression. IBM however has a more insidious course. In this case, subtle proximal weakness was present for three years. Over the subsequent 3-5 years, a more characteristic pattern became apparent. However, at initial presentation, an adult onset slowly progressive proximal lower limb weakness has a broad differential (Table 2). Adult onset myopathies may mimic IBM and vice versa.

In addition to clinical characteristics, muscle biopsy has an important role in differentiating between these diagnoses. In this case, the first biopsy of quadriceps was non-diagnostic and showed only fibrous replacement 
of muscle fibres. A second biopsy of the deltoid was near normal, and only the third biopsy yielded diagnostic features. Carefully selecting an appropriate muscle to biopsy is important in increasing diagnostic yield and preventing repeated procedures. A clinically weak muscle, which is not too severely wasted, is the ideal choice. MRI can be useful in muscle choice. Severely fat infiltrated or atrophic muscle is likely to consist mostly of fibrous or fatty tissue, while an unaffected muscle on MRI is likely to be near normal. Anti-cytosolic 5'nucleotidase $1 \mathrm{~A}$ ( $\mathrm{CN} 1 \mathrm{~A}$, encoded by NT5C1A) have been identified in IBM but testing is still not universally available and diagnostic performance (i.e. sensitivity and specificity) varies widely in the literature.

The classical histological features on a muscle biopsy in IBM are shown in Figure 1. On haematoxylin and eosin (H\&E) stain, myopathic features, endomysial inflammation and eosinophilic inclusions can be seen. Major Histocompatibility Complex class I (MHC I) upregulation and increased number of COX negative fibres can also be seen. Microscopy may demonstrate tubulofilamets ${ }^{17}$. Characteristic rimmed vacuoles may be seen, however $15-20 \%$ of cases may not have rimmed vacuoles ${ }^{18}$. Additional immunohistochemical staining, for example for dystrophin, calpain-3 or myophosphorylase is useful in excluding alternative diagnoses. Congophilic material adjacent to the vacuoles is useful and is part of the diagnostic criteria. Clinicopathological correlation is imperative in guiding this additional staining and interpretation of histology. 


\section{CASE C: An uphill battle.}

A 62 year old male presented with difficulty climbing stairs. He has a past medical history significant for restrictive lung disease, cataracts, eczema and rheumatoid arthritis managed primarily on sulfasalazine. He also has ischaemic heart disease, and prior to symptom onset, had been commenced on Atorvastatin 40mg daily.

He presented with a three year history of progressive difficulty in climbing stairs. Most recently, he had to use the handrail and at times took each step at a time. He also noted difficulty raising his arms above his head. He developed difficulty opening jars, doing buttons and taking his socks off.

At review, it was felt that his rheumatoid arthritis was active. He was then commenced on methotrexate.

On examination, he had normal cranial nerves. Periscapular wasting and bilateral winging was noted as well as more distal upper limb wasting. There was severe proximal weakness, shoulder abduction strength MRC grade 2. Distal weakness was worst at the distal interphalangeal joint (DIP) with MRC grade 3. There was marked quadriceps wasting in the lower limbs with knee extension strength reduced to MRC grade 4. Upper limb reflexes and the knee jerks were reduced or absent. Sensation and co-ordination were normal. The CK was 700 IU/L.

The clinical picture was thought to be atypical for a statin related myopathy, however given the proximity of symptom onset to commencing atorvastatin, it was stopped. Despite this, symptoms continued to worsen.

Given the autoimmune history, myositis was suspected. Myositis-specific antibodies, including antihydroxymethylglutaryl CoA reductase (HMGCR) antibodies were negative. However, a left deltoid muscle biopsy was performed. This muscle was chosen clinically at the time as it was definitely affected by the disease process. The biopsy demonstrated severe atrophy and chronic inflammation.

A subsequent treatment trial with steroids reduced the CK from a range of $600-900$ to $200-400$, however there was no corresponding clinically significant improvement. He went on to have three courses of intravenous immunoglobulin (IVIG) again with no objective clinical improvement.

MRI of lower limb muscles demonstrated severe selective quadriceps involvement. Given the lack of treatment response that would be expected in an (inflammatory) myositis, a repeat muscle biopsy was performed of left 
vastus lateralis which demonstrated mild inflammation, rimmed vacuoles, COX negative fibres, eosinophilic inclusions and p62 positive fibres supportive of a diagnosis of IBM.

\section{Clinical Questions:}

Which condition is a differential diagnosis for severe preferential quadriceps wasting?

1. Fascioscapulohumeral dystrophy

2. Dystrophinopathy

3. Myotonic Dystrophy

4. Becker's Muscular Dystrophy

What is the role for IVIG or other immunomodulators in IBM?

1. There is good Level I evidence for the use of IVIG and immunosuppressive treatment

2. There is no evidence for the use of IVIG and immunosuppressive treatment

3. There is evidence that IVIG is helpful for severe phenotypes

4. There is evidence that methotrexate is helpful for severe phenotypes

Discussion

In this case, the presenting feature was quadriceps involvement, with difficult climbing stairs. He went on to develop quadriceps wasting. Quadriceps involvement is seen characteristically in IBM but can also be seen in other muscle conditions including dystrophinopathies, sarcoglycanopathies and laminopathies ${ }^{19}$. A femoral neuropathy, lumbosacral plexopathy, or L3/4 radiculopathy should not be forgotten, although these are often unilateral and associated with sensory involvement.

In this case, the co-existence of active autoimmune co-morbidities favoured the diagnosis of an immunemediated myositis. There are reports of association of IBM with autoimmune diseases (13-24\%), most commonly Sjogren syndrome (13-24\%). ${ }^{1}$ An association with large granular lymphocytic leukaemia (LGLL) has also been reported. However, there are several aspects that reduced the likelihood of an immune-mediated 
myositis. The onset and progression in this case was more insidious than is often seen in an immune mediated process. The CK is not as high as often seen in immune-mediated myositis, particular in necrotising myositis.

Importantly, there was no objective clinical response to treatment with steroids, methotrexate or IVIG. The evidence to date establishes that IBM is resistant to immunosuppressive treatment ${ }^{17}$. A small retrospective trial demonstrated transient improvement with IVIG only ${ }^{20}$. Clearly defining measures to demonstrate failure to respond to treatment is important. The IBM functional rating scale (IBMFRS), 6-minute walk distance (6MWD) test and timed up and go (TUG) test alongside manual muscle testing are validated assessments that may be helpful. 


\section{Case D: Not what it seems.}

A 69 year old gentleman presented with a 5 year history of progressive lower limb weakness. His CK was 385 IU/L. At initial presentation, lower limb weakness with a subsequent muscle biopsy demonstrating inflammation led to a probable diagnosis of IBM.

The patient was monitored annually. On review in the muscle clinic ten years later, very slow progression was noted. He noted some additional upper limb involvement.

On examination at this stage, shoulder girdle wasting with weak shoulder abduction and external rotation was seen. There was no scapula winging. Distal upper limb strength, including finger flexors, was strong. There was mild weakness in all lower limb muscle groups with marked dorsiflexion weakness with foot drop.

This progression and distribution of weakness was thought to be atypical for IBM, prompting re-investigation. The initial muscle biopsy was re-reviewed with additional immune stains. These stains demonstrated cytoplasmic immune-reactivity in several fibres with p62 and myotillin and less so for desmin. This pattern of staining suggested a myofibrillary myopathy.

Subsequent genetic testing by direct sequencing of exons two to ten of the myotilin (MYOT) gene revealed a pathogenic heterozygous c.179C>G (p.Ser60Cys) variant consistent with a diagnosis of myotilin associated myofibrillar myopathy. Desmin (DES) and FSHD genetic testing were negative.

A diagnosis of myofibrillar myopathy was made, prompting appropriate screening. Cardiac screening revealed a previous silent myocardial infarction.

\section{Clinical Questions:}

Which of the following features is seen in the typical MRI pattern seen in IBM?

1. Relative sparing of rectus femoris

2. Marked fatty infiltration of rectus femoris

3. Marked fatty infiltration of tibialis anterior

4. Relative sparing of quadratus femoris 
Which muscular dystrophy(ies) can have cardiac or respiratory involvement and should not be missed?

1. Oculopharyngeal muscular dystrophy

2. Duchene Muscular Dystrophy

3. Lamin $\mathrm{A} / \mathrm{C}$ deificiency

4. 2 and 3

\section{Discussion}

This case is particularly important in illustrating the need for clinicopathological correlation, which prompted additional staining on the muscle biopsy leading to the ultimate diagnosis.

Muscle MRI is now more readily available and is a useful tool in diagnosis. Characteristic patterns can be seen in muscle conditions which can then direct more targeted genetic testing and biopsy staining. The pattern seen in myotilin associated myofibrillar myopathy is distinct from IBM ${ }^{21}$, Figure 2. In IBM, the typical pattern is fatty replacement, preferentially in the quadriceps femoris, with relative sparing of the rectus femoris and preferential fat accumulation within the medial head of gastrocnemius (in comparison with soleus and lateral gastrocnemius).

Making an accurate diagnosis has important implications in screening for respiratory and cardiac involvement. Myofibrillar myopathies may present with proximal weakness and some muscle biopsy features can cross-over with IBM. Considering this differential and performing appropriate genetic diagnostics is important for an accurate diagnosis and subsequent cardiac and respiratory monitoring as is needed in myofibrillar myopathy. Cardiomyopathy is also seen in Duchenne muscular dystrophy, Emery-Dreifuss, Lamin A/C deficiency, Becker muscular dystrophy, sarcoglycanopathy and dystroglycanopathy. Respiratory impairment is seen in Lamin AC deficiency, sarcoglycanopathy and dystroglycanopathy. 


\section{Case E: Pursuing a diagnosis.}

A 52 year gentleman presented with right knee clicking and giving way. He subsequently underwent right knee arthroscopy. However, over the next two years, skiing became more difficult. He felt the right leg in particular was weak.

This progressed slowly and he found that he could no longer run and he had difficulty with tasks such as climbing stairs carrying a heavy load and going downstairs. Getting off the floor from lying was increasingly difficult. The slow progression and distribution of weakness made the diagnosis of IBM likely.

EMG was non-diagnostic and muscle biopsy was arranged. Biopsy of right vastus lateralis demonstrated myopathic features with some inflammatory components including MHC class 1 upregulation, all seen in IBM. However, no vacuolation or eosinophilic inclusions were seen. Additionally, prominent lobulated and ring fibres, while non-specific, suggested genetic myopathies including Muscular dystrophy (type 2 - DM2).

Additional tests including a CK of $356 \mathrm{IU} / \mathrm{L}$ and slow progression continued to remain consistent with probable IBM. However, given some atypical muscle biopsy changes, genetic testing was pursued. Testing for the CNBP expansion mutation seen in DM2 was negative.

An MRI of the limbs was performed which demonstrated asymmetrical thigh involvement with fat replacement. Clinically, the upper limbs were normal with no evidence of wasting, however upper limb MRI demonstrated reduced bulk and fatty signal in the right trapezius and left serratus anterior (Figure 3). This pattern of muscle involvement on MRI and marked asymmetry prompted investigation for FSHD.

FSH genetic testing returned positive with a partial deletion of the D4Z4 4q35 repeat region with a $31 \mathrm{~kb}$ fragment demonstrated (pathogenic $<39 \mathrm{~kb}$ ). Given the patient's mild/atypical clinical phenotype second-line FSHD testing was requested, namely permissive haplotype analysis. Further genetic testing using the $4 \mathrm{qA}$ probe revealed that the above 31-kb fragment was associated with the 4qA haplotype in this patient.

\section{Clinical Questions?}

Which diagnosis can also demonstrates inflammatory features on muscle biopsy? 
1. FSHD

2. Myotonia Congenita

3. SMA

4. Motor Neurone Disease

\section{Discussion}

Myopathic muscle biopsies with inflammatory features can be seen in several conditions beyond classical myositis (idiopathic inflammatory myopathies). It is important to consider other myopathies when faced with a biopsy with inflammatory cells. Up to a third of muscle biopsies in FSHD can have an inflammatory infiltrate ${ }^{18}$. The infiltrate is composed of CD4+ and CD8+ lymphocytes, however invasion of intact muscle fibres is rarely seen. In dysferlinopathies, up to a third can have an inflammatory infiltrate predominantly of T cells and macrophages. A small number of calpainopathies can have an eosinophilic infiltrate.

In this case, the clinical picture was informative and reviewing the phenotype over the disease course was very helpful. The upper limb involvement demonstrated on MRI and marked lower limb asymmetry, prompted investigation for conditions that more classically demonstrate this pattern of muscle involvement. In this case, again neuromuscular MRI was very useful in detecting patterns of involvement. 


\section{Summary}

We describe three cases with atypical or challenging aspects in diagnosing IBM as well as two cases of inherited myopathies that may mimic IBM. Atypical presentations, such as dysphagia, may prompt investigation for alternative aetiologies, however IBM should also be considered in the differential diagnosis. Specifically examining and imaging other characteristically affected muscle groups may aid diagnosis.

The presence of inflammation on muscle biopsy is an important aspect to discuss. Inflammatory features are seen in conditions beyond dermatomyositis, polymyositis and immune-mediated necrotising myopathy. Similar inflammatory features can be seen in IBM and also FSHD which should be considered in the differential diagnosis. Clinico-pathological correlation in multi-disciplinary meetings are very helpful in interpreting biopsy changes in clinical context and guiding further biopsy staining. Treatment resistance to immunosuppression should additionally prompt investigation for IBM.

MRI can be an extremely helpful tool in accurate diagnosis. As illustrated, specific patterns can be seen in IBM compared to other inherited myopathies. MRI is also useful in selection of muscle to biopsy.

Clinical characteristics, appropriate muscle biopsy with clinically guided staining and neuromuscular MRI patterns, in combination are likely to improve diagnostic rates in IBM and its mimics. 


\section{References}

1. Greenberg SA. Inclusion body myositis: clinical features and pathogenesis. Nat Rev Rheumatol. 2019;15(5):257-272. doi:10.1038/s41584-019-0186-x

2. Callan A, Capkun G, Vasanthaprasad V, Freitas R, Needham M. A Systematic Review and Meta-Analysis of Prevalence Studies of Sporadic Inclusion Body Myositis. J Neuromuscul Dis. 2017;4(2):127-137. doi:10.3233/JND-160198

3. Badrising UA, Maat-Schieman MLC, Van Houwelingen JC, et al. Inclusion body myositis: Clinical features and clinical course of the disease in 64 patients. J Neurol. 2005;252(12):1448-1454. doi:10.1007/s00415-005-0884-y

4. Keshishian A, Greenberg SA, Agashivala N, Baser O, Johnson K. Health care costs and comorbidities for patients with inclusion body myositis. Curr Med Res Opin. 2018;34(9):1679-1685. doi:10.1080/03007995.2018.1486294

5. Capkun G, Schmidt J, Ghosh S, et al. Development and validation of a Bayesian survival model for inclusion body myositis. Theor Biol Med Model. 2019;16(1):1-10. doi:10.1186/s12976-019-0114-4

6. Mendell JR, Sahenk Z, Al-Zaidy S, et al. Follistatin Gene Therapy for Sporadic Inclusion Body Myositis Improves Functional Outcomes. Mol Ther. 2017;25(4):870-879. doi:10.1016/j.ymthe.2017.02.015

7. Hanna MG, Badrising UA, Benveniste O, et al. Safety and efficacy of intravenous bimagrumab in inclusion body myositis (RESILIENT): a randomised, double-blind, placebo-controlled phase $2 \mathrm{~b}$ trial. Lancet Neurol. 2019;18(9):834-844. doi:10.1016/S1474-4422(19)30200-5

8. Ahmed M, MacHado PM, Miller A, et al. Targeting protein homeostasis in sporadic inclusion body myositis. Sci Transl Med. 2016;8(331):28-31. doi:10.1126/scitransImed.aad4583

9. Benveniste O, Hogrel J-Y, Annoussamy M, et al. Rapamycin Vs. Placebo for the Treatment of Inclusion Body Myositis: Improvement of the 6 Min Walking Distance, a Functional Scale, the FVC and Muscle $\begin{array}{llll}\text { Quantitative } \quad \text { MRI } \quad \text { [abstract]. ACR/ARHP Annu } & \text { Meet. }\end{array}$ http://www.myositis.org/storage/documents/IBM_Published_Research/Rapamycin_Vs_Placebo_for_t he_Treatment_of_Inclusion_Body_Myositis_ACR_abstract_2017.pdf. 
10. Langdon PC, Mulcahy K, Shepherd KL, Low VH, Mastaglia FL. Pharyngeal dysphagia in inflammatory muscle diseases resulting from impaired suprahyoid musculature. Dysphagia. 2012;27(3):408-417. doi:10.1007/s00455-011-9384-7

11. Benveniste O, Guiguet M, Freebody J, et al. Long-term observational study of sporadic inclusion body myositis. Brain. 2011;134(11):3176-3184. doi:10.1093/brain/awr213

12. Cortese A, Machado P, Morrow J, et al. Longitudinal observational study of sporadic inclusion body myositis: Implications for clinical trials. Neuromuscul Disord. 2013;23(5):404-412. doi:10.1016/j.nmd.2013.02.010

13. Cox FM, Titulaer MJ, Sont JK, Wintzen AR, Verschuuren JJGM, Badrising UA. A 12-year follow-up in sporadic inclusion body myositis: An end stage with major disabilities. Brain. 2011;134(11):3167-3175. doi:10.1093/brain/awr217

14. Benveniste $\mathrm{O}$, Guiguet $\mathrm{M}$, Freebody J, et al. Long-term observational study of sporadic inclusion body myositis. Brain. 2011;134(11):3176-3184. doi:10.1093/brain/awr213

15. Mulcahy KP, Langdon PC, Mastaglia F. Dysphagia in inflammatory myopathy: Self-report, incidence, and prevalence. Dysphagia. 2012;27(1):64-69. doi:10.1007/s00455-011-9338-0

16. Oh TH, Brumfield KA, Hoskin TL, Kasperbauer JL, Basford JR. Dysphagia in inclusion body myositis. Am J Phys Med Rehabil. 2008;87(11):883-889. doi:10.1097/PHM.0b013e31818a50e2

17. Machado P, Brady S, Hanna MG. Update in inclusion body myositis. Curr Opin Rheumatol. 2013;25(6):763-771. doi:10.1097/01.bor.0000434671.77891.9a

18. Michelle EH, Mammen AL. Myositis Mimics. Curr Rheumatol Rep. 2015;17(10):1-8. doi:10.1007/s11926-015-0541-0

19. Mercuri E, Muntoni F. Muscular dystrophies. Lancet (London, England). 2013;381(9869):845-860. doi:10.1016/S0140-6736(12)61897-2

20. Dobloug C, Walle-Hansen R, Gran JT, Molberg. Long-term follow-up of sporadic inclusion body myositis treated with intravenous immunoglobulin: A retrospective study of 16 patients. Clin Exp Rheumatol. 
2012;30(6):838-842.

21. Bugiardini E, Morrow JM, Shah S, et al. The diagnostic value of MRI pattern recognition in distal myopathies. Front Neurol. 2018;9(JUN):1-11. doi:10.3389/fneur.2018.00456 